University of Wollongong

Research Online

Faculty of Social Sciences - Papers (Archive) Faculty of Arts, Social Sciences \& Humanities

2015

\title{
Walking without optic flow reduces subsequent vection
}

Takeharu Seno

Kyushu University, senosann@gmail.com

Stephen Palmisano

University of Wollongong, stephenp@uow.edu.au

B Riecke

Simon Fraser University

S Nakamura

Nihon Fukushi University

Follow this and additional works at: https://ro.uow.edu.au/sspapers

Part of the Education Commons, and the Social and Behavioral Sciences Commons

Research Online is the open access institutional repository for the University of Wollongong. For further information contact the UOW Library: research-pubs@uow.edu.au 


\title{
Walking without optic flow reduces subsequent vection
}

\begin{abstract}
This experiment investigated the effect of walking without optic flow on subsequent vection induction and strength. Two groups of participants walked for $5 \mathrm{~min}$ (either wearing Ganzfeld goggles or with normal vision) prior to exposure to a vection-inducing stimulus. We then measured the onset latency and strength of vection induced by a radially expanding pattern of optic flow. The results showed that walking without optic flow transiently yielded later vection onsets and reduced vection strength. We propose that walking without optic flow triggered a sensory readjustment, which reduced the ability of optic flow to induce self-motion perception.
\end{abstract}

\section{Keywords}

subsequent, reduces, vection, flow, walking, optic, without

Disciplines

Education | Social and Behavioral Sciences

\section{Publication Details}

Seno, T., Palmisano, S., Riecke, B. E. \& Nakamura, S. (2015). Walking without optic flow reduces subsequent vection. Experimental Brain Research, 233 (1), 275-281. 
Walking without optic flow reduces subsequent vection

Takeharu Seno*+, Stephen Palmisano**, Bernhard E. Riecke*** \& Shinji Nakamura****

1. Institute for Advanced Study, Kyushu University

6-10-1 Hakozaki, Higashi-Ku, Fukuoka, 812-8581, Japan

2. Faculty of Design, Kyushu University

4-9-1 Shionaru, Minami-ku, Fukuoka, Japan

3. Research Center for Applied Perceptual Science, Kyushu University seno@design.kyushu-u.ac.jp

**S School of Psychology, University of Wollongong, NSW, Australia, 2522

*** School of Interactive Arts and Technology (SIAT), Simon Fraser University, 250-13450 102nd Avenue, Surrey, BC, V3T 0A3 Canada

**** Nihon Fukushi University, Faculty of Child Development, Division of Clinical Psychology, Okuda, Mihama-cho, Aichi 470-3295, Japan

+ Corresponding author: Takeharu Seno

Kyushu University

Email: seno@design.kyushu-u.ac.jp

TEL: +81-80-3538-8374 


\begin{abstract}
This experiment investigated the effect of walking without optic flow on subsequent vection induction and strength. Two groups of participants walked for 5 minutes (either wearing Ganzfeld goggles or with normal vision) prior to exposure to a vection-inducing stimulus. We then measured the onset latency and strength of vection induced by a radially expanding pattern of optic flow. The results showed that walking without optic flow transiently yielded later vection onsets and reduced vection strength. We propose that walking without optic flow triggered a sensory readjustment, which reduced the ability of optic flow to induce self-motion perception.
\end{abstract}

Key words,

Vection, self-motion perception, adaptation, sensory readjustment 


\section{Introduction}

Multiple senses contribute to the perception of self-motion, including vision, the vestibular system of the inner ear, the proprioceptive estimation of limb/joint movement and position, the somatosensory system of cutaneous receptors and even audition (Gibson 1966; Howard 1982). While the inputs of these different "self-motion" senses appear to be integrated (Rieser et al. 1995), vision is thought to play a particularly important role in the perception of self-motion (see Dichgans and Brandt 1978; Howard 1982; Riecke 2011). In fact, exposure to a visual motion field that mimics the retinal flow produced by locomotion typically induces a compelling illusion of self-motion (referred to as 'vection' - Fischer and Kornmüller 1930). For example, when a train begins to move out from the station, it is common for stationary observers nearby to misperceive that they themselves are in motion (rather than the train - Seno and Fukuda 2012).

A number of recent studies suggest that such visually-mediated self-motion perceptions can be facilitated by physically moving the observer in a manner consistent with the visual simulation (Berger et al. 2010; Wong and Frost 1981; Wright, 2009; Bubka and Bonato 2010) or by incorporating active head motions of the observer directly into the self-motion display (Ash et al. 2011a; Ash et al. 2011b). ${ }^{1}$ When taken together, such findings suggest that consistent multisensory stimulation may produce a more compelling overall experience of self-motion than visual self-motion stimulation alone.

But what happens when the multisensory patterns of self-motion stimulation are either inconsistent with each other or atypical based on past

\footnotetext{
${ }^{1}$ There are, however, examples where consistent cross-modal stimulation does not enhance but rather reduce vection: For example adding velocity-matched linear treadmill walking to a visual forward motion simulation has been shown to reduce vection (Ash, Palmisano, \& Allison, 2012; Kitazaki, Onimaru, \& Sato, 2010; Onimaru, Sato, \& Kitazaki, 2010), whereas linear treadmill walking was found to enhance vection when the visual velocity was 30 times faster than the walking velocity (Seno, Ito, \& Sunaga, 2011). Interestingly, however, velocity-matched circular treadmill walking appears to enhance visually induced circular vection (Freiberg, Grechkin, \& Riecke, 2013; Riecke, Freiberg, \& Grechkin, 2014).
} 
experience? Given the vection enhancements outlined above during consistent multisensory stimulation conditions, one might expect to see large vection impairments. However, several recent studies appear to show that visually induced vection is surprisingly tolerant to a number of so-called "sensory conflict" situations (Ash and Palmisano 2012; Ash et al. 2011a; Ash et al. 2011b; Kim and Palmisano 2008, 2010; Palmisano et al. 2011). For example, we have found that compelling vection can still be induced even when visual and non-visual self-motion stimulations are 180 degrees out-of-phase or indicate self-motion along completely different axes (Ash and Palmisano 2012). One possible explanation for such findings is that during prolonged exposure to these types of 'sensory conflict' conditions, the brain may engage in some sort of sensory/multisensory readjustment in order to minimize the (assumed) conflicts between the different self-motion senses.

While sensory/multisensory readjustment could potentially explain the findings of many laboratory-based self-motion studies, there has typically been no objective evidence as to whether or not such a sensory readjustment of the relationship between the visual and non-visual cues to self-motion actually occurred. In the past, several investigators have searched for an objective index for such sensory changes. However, it has proven difficult to find. In one such study, Harris, Morgan and Still (1981) proposed that visual motion aftereffects (MAEs) might provide an objective index of sensory recalibration. MAEs refer to the illusory motion of a physically stationary scene which is experienced after prolonged exposure to sustained visual movement. In the Harris et al study, participants viewed optic flow displays simulating self-motion in depth while either stationary or seated on a trolley that moved during the display. The trolley motions either generated consistent or inconsistent multisensory self-motion stimulation (i.e. the trolley moved in the same or the opposite direction to the visually simulated self-motion). Harris and colleagues predicted that if the brain recalibrates during sensory conflict, and if MAEs serve as an index of this sensory recalibration, then stationary conditions and inconsistent trolley motions should produce stronger visual MAEs than consistent trolley 
motions. However, only partial support was found for these hypotheses. Consistent trolley motions were found to strongly suppress the MAEs generated by forward simulated self-motions (compared to those generated during stationary viewing). However, consistent trolley motions did not significantly suppress the MAEs generated by simulated backwards self-motion. Furthermore, these MAEs were not enhanced by putting the visually simulated and trolley based self-motions into conflict. Since similar patterns of results had previously been found by Wallach and Flaherty (1975), these findings weaken the case for a MAE-based index of sensory recalibration.

Based on previous findings of surprisingly compelling vection during situations of (assumed) sensory conflict, we hypothesized that the nature of the vection experience ${ }^{2}$ depends on: (1) relative influence of visual inputs in the multisensory processing of self-motion perception; and (2) that this influence might be reduced (compared to that of the non-visual senses) by prolonged exposure to self-motion without optic flow.

To investigate this idea, we compared the vection induced directly after 5 -minutes of walking either with normal vision or while wearing Ganzfeld goggles that removed all visual flow without affecting overall luminance. We hypothesized that compared to the control condition, optic flow deprivation during the Ganzfeld walking condition should lead to a reduction in participants' susceptibility to vection, which (if it was due to sensory readjustment) should fade quickly following repeated exposure to the optic flow.

\section{Method}

\section{Ethics statement}

\footnotetext{
2 In this article we used term "vection" as "visually induced" self-motion perception in the absence of physical self-motion.
} 
Our experiments were pre-approved by the Ethics Committee of Kyushu University, and informed verbal consent was obtained from each participant prior to testing.

\section{Participants}

Twenty-five volunteers participated in this experiment. Participants included both graduate and undergraduate students, as well as assistant professors (they were 14 males and 11 females ranging in age from 21 to 45 years). All participants reported normal vision and no history of vestibular system diseases. None of them were aware of the purpose of the experiment, although all had previously participated in vection experiments. Participants were randomly divided into two groups (Ganzfeld and Control conditions): Twelve participants ( 7 males, 5 females; mean age 24.3) were assigned to the Ganzfeld condition and thirteen participants (7 Males, 6 females; mean age 26.9) were assigned to the Control condition.

\section{Stimuli}

The vection inducing stimuli were generated and controlled by a computer (Apple MacBook Pro) and presented on a plasma display (3D Viera 70 inch, Panasonic, Japan) with $1,024 \times 768$ pixel resolution at a $60 \mathrm{~Hz}$ refresh rate, presented without stereo mode. The display showed radially expanding patterns of optic flow simulating forward self-motion at $20 \mathrm{~m} / \mathrm{s}$ (simulated display depth was $20 \mathrm{~m}$ ). As the dots in these displays disappeared off the edge of the screen, they were replaced at the far depth plane, thereby creating an endless optic flow display. Approximately 1,240 dots were presented in each frame. Each dot was of constant size on the screen and subtended a visual angle of $0.03^{\circ}-0.05^{\circ}$, depending on its eccentricity on the screen. As these moving dots patterns did not form a density gradient, they did not provide static depth cues. Thus, motion perspective was the only depth cue provided. There was no fixation point, but participants were asked to look at the centre of the optical expansion. The viewing distance was 57 
$\mathrm{cm}$, yielding a visual field of view of $100^{\circ} \times 72^{\circ}$.

\section{Procedure}

\section{Apparatus}

Two conditions were tested in this between-subjects designed experiment: a walking condition with Ganzfeld viewing (Ganzfeld), and a normal viewing walking condition (Control). The Ganzfeld goggles were constructed from two ping-pong balls (sliced in half) and attached to a metal frame. These goggles prevented the participant from seeing any details of the outside world (he/she only saw a blank bright field without any specific visual features - Figure 1). The Ganzfeld condition was used (rather than a blindfold) to avoid very different dark adaptation. Even though there were luminance differences, these were would have been minimal.

Participants initially walked for 5 minutes around the ground floor of the Kyushu University building in which the experimental vection testing chamber was located. The room for the walking was normally lit. Participants in both the Ganzfeld and the Control conditions walked quite slowly $(<1 \mathrm{~m} / \mathrm{sec}$ on average) and were always accompanied by the experimenter. As illustrated in Figure 1, the participant and the experimenter held opposite ends of the same $25 \mathrm{~cm}$ long wooden bar, which was used by the experimenter to lead the participants safely when walking in Ganzfeld conditions, and to control walking speeds in both conditions ${ }^{3}$.

At the end of this 5 minute walking period, participants in both the Ganzfeld and Control conditions were directly led to the dark experimental vection testing chamber and seated in front of the visual self-motion display. They

\footnotetext{
${ }^{3}$ Even though the experimenter endeavored to have participants walk at the same pace in both conditions, participants were (not surprisingly) somewhat more cautious when walking during Ganzfeld conditions.
} 
were then immediately exposed (sequentially) to four vection-inducing trials - on each trial of these trials they were presented with a computer generated visual self-motion display for $30 \mathrm{~s}$. In the Ganzfeld condition, participants wore the goggles until just before the optic flow stimulus presentation. Note that in order to avoid any potential context-specific influences, the Ganzfeld and Control conditions were conducted in the same corridor and lighting conditions during the walking phase and the same darkened test room and stimuli for the vection testing.
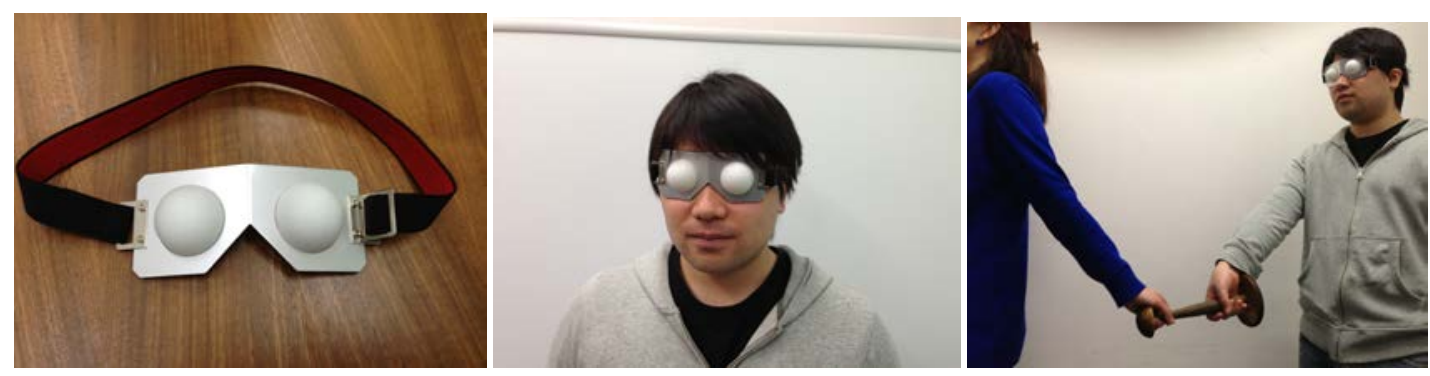

Figure 1. The translucent Ganzfeld goggles used in this experiment and an illustration of how the experimenter used a wooden bar to guide participants.

When viewing the computer generated radially expanding pattern of optic flow, participants were asked to press a designated button as soon as they perceived forward self-motion. After each trial, the participants rated the subjective vection strength using a 101-point rating scale ranging from 0 (no self-motion was perceived) to 100 (very strong perceived self-motion). Immediately after the observer's verbal response, the visual stimulus for next trial was presented on the screen, i.e., there was no rest period between the four trials.

\section{Results}




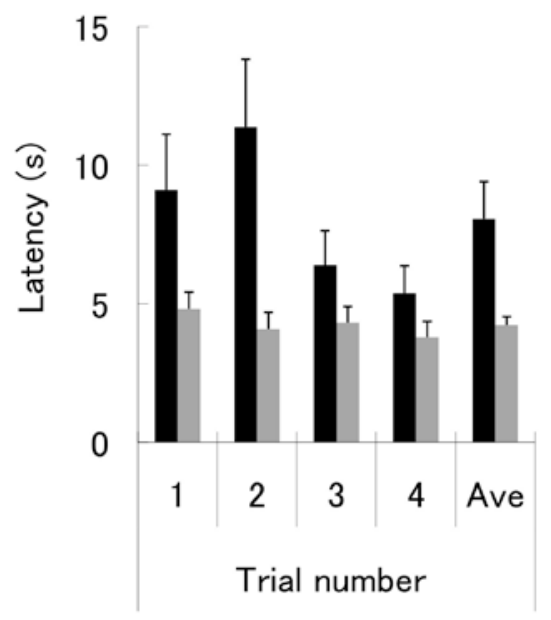

(a)

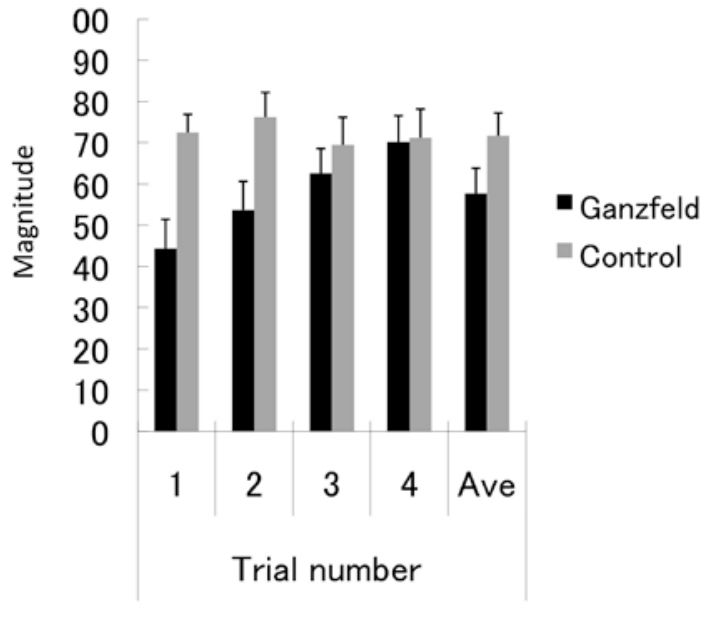

(b)

Figure 2. Mean vection latency (a) and magnitude (b) for each of the four trials per person. The black and grey bars indicate the Ganzfeld and the Control conditions, respectively. Error bars indicate standard errors. "Ave" indicates the average values over the four trials.

We measured both vection onset latency and verbal ratings of vection strength. The vection onset latency was the time taken from the start of exposure to the optic flow until the participant's button press. Stronger vection tends to have both shorter onset latencies and higher estimated magnitudes. ${ }^{4}$

Figure 2 shows the two vection measures (onset and strength - averaged across participants) as a function of the vection trial for the two different walking conditions (Ganzfeld vs. Control) ${ }^{5}$. Split-plot ANOVAs (Two walking conditions [between-subjects factor: Ganzfeld vs Control] and four trial repetitions [within-subjects factor]) revealed significant main effects of walking condition on both the vection latency, $F(1,22)=7.49, p=.01, \eta_{\mathrm{G}}^{2}$

\footnotetext{
4 We should note here that there were some vection drop-outs during the stimulus presentation. During the stimulus presentation period, participants sometimes did have a period without vectuion at all.

5 The latency data for one trial was lost for one of our participants (his initial was TM) in the Control condition. We excluded latency data obtained from such participant from subsequent alanyses.
} 
$=.16$, and vection strength data, $F(1,23)=81.84, p<.0001, \eta_{\mathrm{G}}^{2}=.75$. These ANOVAs also revealed a significant main effect of trial repetition on vection latency, $F(3,66)=4,07, p=.01, \eta_{\mathrm{G}}^{2}=.08$, but not on vection strength, $F(3,69)$ $=.92, p=.43, \eta_{\mathrm{G}}^{2}=.01$. Interactions between these factors were also found to be significant for both latency, $F(3,66)=3.00, p=.04, \eta_{\mathrm{G}}^{2}=.06$, and magnitude, $F(3,69)=3.02, p=.04, \eta_{\mathrm{G}}^{2}=.02$. Arguably, these significant interactions must be the most important result, we think.

Vection occurred later and was rated as being weaker in the Ganzfeld condition compared to the Control condition. That is, vection was reduced by a 5-minute period of physical walking without accompanying visual self-motion stimulation just before the display presentation (at least when compared to vection in the Control condition). Figure 2 shows that the effect of this optic flow deprivation during walking on subsequent vection induction was larger in the first two trials (trials $1 \& 2$ ), with this effects diminishing in the latter two trials (trials $3 \& 4$ ). That is, the first two trials yielded later vection onsets and reduced vection strength than trials 3 and 4 .

Below we summarize the results from the multiple comparisons (Ryan's method, and the significance level was $5 \%$ ). In the Control condition, there were no significant differences in either latency or vection strength ratings across the four trials. By contrast, in the Ganzfeld condition, significant differences were found between the first two trials (trials $1 \& 2$ ) and the last two trials (trials $3 \& 4$ ) in both vection latency and vection strength. There were also significant differences between the Control and Ganzfeld conditions in the first and second trials in terms of both vection latency and magnitude. There were no significant differences between the Control and Ganzfeld conditions in the third and fourth trials for any of the vection indices.

The effect sizes were larger in vection magnitude than in vection latency. This might be related to the fact that the changes in vection are easier to observer in terms of strength rather than in latency. In our previous studies, 
the vection strength was most reliable and sensitive measure of the changes of vection (e.g. Seno et al. 2013).

\section{Discussion}

Prolonged periods of physical self-motion without corresponding visual motion (Ganzfeld conditions) were found to strongly reduce the ability of optic flow to subsequently induce vection (compared to Control conditions which provided consistent visual and non-visual information about self-motion). Specifically, five minutes optic flow deprivation while walking was sufficient to strongly reduce the vection induced in the first and second vection test trials, indicated by delayed vection onset and reduced vection strength. Importantly, both types of inhibitory ${ }^{6}$ vection effects were rather short-lived and lasted only for two trials. No significant differences were found between the Ganzfeld and Control walking conditions on either vection measure during the third and fourth test trials.

As indicated in the introduction, multiple sensory systems are known to be involved in self-motion perception. Here, we proposed that prolonged self-motion stimulation without optic flow might temporarily decrease the influence of visual (compared to non-visual) self-motion inputs in this multisensory integration process. The vection reductions observed in the Ganzfeld (compared to the Control) walking conditions are consistent with a sensory (or possibly even a multisensory) readjustment, which favored the non-visual self-motion inputs and/or suppressed the visual self-motion inputs. Since the self-motion illusions examined in this experiment were purely visually induced (i.e. they were experienced by physically stationary observers), when the relative influence of visual inputs were decreased, vection induction should have been inhibited as well, which is exactly what

\footnotetext{
${ }^{6}$ While it is possible that prior walking with optic flow facilitated subsequent vection, it is more likely that prior walking without optic flow inhibited vection induction. However, in order to determine whether this was indeed the case, we would need a control condition where the participant was stationary for 5 minutes prior to exposure to the optic flow.
} 
we observed (i.e. increased vection onset latencies and reduced vection strength ratings). The assumption was that these effects had a cortical origin. However, the cortical mechanisms underlying these transient effects are unclear - they could in principle have arisen via habituation, adaptation, sensory reweighting or sensory recalibration.

Importantly, we also predicted that if a sensory readjustment was responsible for the above effects, then the Ganzfeld walking conditions should only temporarily inhibit vection ${ }^{7}$. Consistent with this prediction, the recovery of vection following Ganzfeld walking can be clearly seen over the course of the four successive test trials on both vection measures (these measures were not statistically different for Ganzfeld and Control conditions on the third and fourth testing trials). Since the time taken to complete each vection test trial was about 1 minute (which includes not only the 30 seconds exposure to the optic flow display, but also the time taken for the participant to make their overall vection strength response for the trial, and the interstimulus interval), vection recovery for both measures appeared complete only 2 minutes after Ganzfeld walking. It appears thus that any sensory readjustment generated by the Ganzfeld walking was quite short-lived, at least in the case of five minute adaptation.

In the introduction we proposed that sensory readjustment is likely to occur when we are selectively deprived of the information provided by one or more of self-motion senses. Intriguingly, it is not just the Ganzfeld walking conditions (Walking without optic flow), but also the vection testing conditions (Optic flow without walking) in the current experiment that meet this criterion for sensory readjustment. However, we expected sensory readjustments to occur in opposite directions in these different situations -

\footnotetext{
${ }^{7}$ If there was a perceptual effect of the Ganzfeld viewing on vection we would have expected it to be transient. It is possible however that if there had instead been a cognitive or experimental demand based effect of Ganzfeld viewing then this might have been more likely to be long (or longer) lasting.
} 
the former case should favour non-visual self-motion inputs, whereas the latter case should favour visual self-motion inputs. It was therefore possible that the vection recovery from Ganzfeld walking seen in the third and fourth testing trials might also (in part at least) reflect the occurrence of a second sensory readjustment process - this time favouring the visual-only self-motion testing conditions. However, as can be seen in Figure 2, trial-based vection improvements were only seen in the Ganzfeld walking conditions (not in the Control conditions), which suggests that our four $30 \mathrm{~s}$ long vection testing conditions were not sufficient to initiate their own sensory readjustment process.

Self-motion perception is primarily a multisensory experience (e.g. Gibson 1966; Rieser et al. 1995; Seno et al. 2011; Allison et al. 2012; Riecke and Schulte-Pelkum 2013). While vection is often considered a purely visual illusion of self-motion, one cannot hope to fully understand self-motion perception by examining the role that vision plays in it alone. It is important to also examine the consequences of providing consistent and inconsistent multisensory self-motion stimulations ${ }^{8}$. Past research has shown that the vection experience can be facilitated, inhibited, or unaffected by these different types of multisensory self-motion stimulation. Unfortunately, it is difficult to directly investigate multisensory processing underlying these self-motion perceptions at the cortical level (since the observers in brain imaging studies are by necessity always physically stationary - e.g. Pitzalis et al. 2013). Here we report perceptual/behavioural evidence (i.e. not based on brain imaging) that strongly supports of the notion that sensory readjustment occurs during prolonged adaptation to unusual/inconsistent patterns of multisensory self-motion stimulation.

\footnotetext{
8 It should also be noted here that we believe that our results were not a result of dark adaptation but were mediated by sensory readjustment as we hypothesized. In future we should also examine potential effects of walking with eye closed or walking in the complete dark room.
} 
Since we did not have access to an objective index of sensory readjustment and were concerned with carry-over between the Ganzfeld and control condition, we deliberately chose to use a between-subjects (as opposed to within-subjects) design for this experiment. Since participants were only ever exposed to one of the different adaptation conditions (Ganzfeld or Control) and were not aware of the other condition, using a between-subjects design has eliminated possible carry-over effects between conditions and therefore has minimized the likelihood of either their cognitions or any experimental demands influencing their vection experience.

What are the implications of the current findings? In many "real world" situations, the visual and non-visual senses are thought to provide consistent information about self-motion, and thus the integration of this information presumably occurs in a straight-forward fashion. However, in other situations, such as driving an automobile along a straight expressway for an extended period, the self-motion perception may be predominantly determined by the available visual information. In such situations, it would seem likely there will be a modulation in driver's self-motion perception (before and after driving), because the current study appears to show that atypical combinations of self-motion sensations trigger sensory readjustments, which can (at least transiently) affect subsequent self-motion perceptions.

\section{Conclusion}

This study suggests that sensory readjustment occurs when observers walk without any exposure to optic flow. We propose that the relative influence of visual self-motion inputs was reduced (presumably at the cortical level) in these conditions compared to normal walking conditions, the result being that vection induction and strength are strongly, but only temporarily, inhibited. These findings confirm that vection is mediated by a multisensory integration process, which can be significantly affected by prior sensory readjustment. 


\section{References}

Allison RS, Zacher JE, Kirollos R, Guterman PS, Palmisano S (2012) Perception of smooth and perturbed vection in short-duration microgravity. Experimental Brain Research 223:479-487 doi: 10.1007/s00221-012-3275-5

Ash A, Palmisano S, Apthorp D, Allison RS (2013) Vection in depth during treadmill walking. Perception 42:562-76. doi:10.1068/p7449

Ash A, Palmisano S (2012) Vection during conflicting multisensory information about the axis, magnitude, and direction of self-motion. Perception 41:253-267. doi:10.1068/p7129

Ash A, Palmisano S, Govan DG, Kim J (2011a) Display lag and gain effects on vection experienced by active observers. Aviation, Space, and Environmental Medicine 82.763-769 doi:10.3357/ASEM.3026.2011

Ash A, Palmisano S, Kim J (2011b) Vection in depth during consistent and inconsistent multisensory stimulation. Perception 40:155-174 doi:10.1068/p6837

Ash A, Palmisano S, Allison R (2012) Vection in depth during treadmill locomotion. Journal of Vision 12:181

Berger DR, Schulte-Pelkum J, Bülthoff HH (2010) Simulating believable forward accelerations on a stewart motion platform. ACM Trans. Appl. Percept 7.1-27 doi:10.1145/1658349.1658354

Bubka A, Bonato F (2010) Natural visual-field features enhance vection. Perception 39:627-635 doi:10.1068/p6315

Dichgans J, Brandt T (1978) Visual-Vestibular Interaction: Effects on Self-Motion Perception and Postural Control. Perception, Handbook of Sensory Physiology (Vol. VIII, pp. 756 - 804). Springer.

Feuereissen D (2013, August). Self-motion illusions (vection) in Virtual Environments: Do active control and user- generated motion cueing enhance visually induced vection? (MSc Thesis). Surrey, BC, Canada: Simon Fraser University. Retrieved from https://theses.lib.sfu.ca/thesis/etd7976

Fischer MH, Kornmüller AE (1930) Optokinetisch ausgelöste 
Bewegungswahrnehmung und optokinetischer Nystagmus [Optokinetically induced motion perception and optokinetic nystagmus]. Journal für Psychologie und Neurologie 273-308.

Freiberg J, Grechkin T, Riecke BE (2013) Do walking motions enhance visually induced self-motion illusions in virtual reality? IEEE Virtual Reality. Orlando, FL, USA: IEEE.

Gibson JJ (1966) The Senses Considered as Perceptual Systems. Houghton Mifflin, Boston, MA.

Harris LR, Morgan MJ, Still AW (1981) Moving and the motion after-effect. Nature 293:139-141 doi: 10.1038/293139a0

Howard IP (1982) Human visual orientation. Chichester, New York, Engl.: J. Wiley.

Keshavarz B, Hettinger LJ, Vena D, Campos JL (2014) Combined effects of auditory and visual cues on the perception of vection. Experimental Brain Research 232:827-836 doi:10.1007/s00221-013-3793-9

Kim J, Palmisano S (2008) Effects of active and passive viewpoint jitter on vection. Brain Research Bulletin 77:335-342 doi: 10.1016/j.brainresbull.2008.09.011.

Kim J, Palmisano S (2010) Visually mediated eye movements regulate the capture of optic flow in self-motion perception. Experimental Brain Research 202:355-361 doi: 10.1007/s00221-009-2137-2.

Kitazaki M, Onimaru S, Sato T (2010) Vection and Action are Incompatible (pp. 22-23). Presented at the 2nd IEEE VR 2010 Workshop on Perveptual Illusions in Virtual Environments (PIVE), Waltham, MA, USA.

Onimaru S, Sato T, Kitazaki M.(2010) Veridical walking inhibits vection perception. Journal of Vision 10:860. doi:10.1167/10.7.860

Palmisano S, Allison RS, Kim J, Bonato F (2011) Simulated viewpoint jitter shakes sensory conflict accounts of vection. Seeing and Perceiving 24:173-200 doi:10.1163/187847511X570817

Pitzalis S, Sdoia S, Bultrini A, Committeri G, Russo FD, Fattori P, Galletti C, Galati G (2013) Selectivity to translational egomotion in human brain motion areas. PLoS ONE 8:e60241. doi: 10.1371/journal.pone.0060241 
Riecke BE (2011) Compelling Self-Motion Through Virtual Environments Without Actual Self-Motion - Using Self-Motion Illusions ("Vection") to Improve User Experience in VR. In J. Kim (Ed.). In J.-J. Kim (Ed.), Virtual Reality (pp. 149-176). InTech. Retrieved from http://www.intechopen.com/articles/show/title/compelling-self-motionthrough-virtual-environments-without-actual-self-motion-using-selfmotion-ill

Riecke BE, Freiberg J, Grechkin TY (submitted). Can Walking Motions Improve Visually Induced Rotational Self-Motion Illusions in Virtual Reality? Journal of Vision.

Riecke BE, Schulte-Pelkum J (2013) Perceptual and Cognitive Factors for Self-Motion Simulation in Virtual Environments: How Can Self-Motion Illusions ("Vection") Be Utilized? In F. Steinicke, Y. Visell, J. Campos, \& A. Lécuyer (Eds.), Human Walking in Virtual Environments (pp. 27-54). New York: Springer.

Riecke BE, Schulte-Pelkum J, Caniard F, Bülthoff HH (2005a) Influence of Auditory Cues on the visually-induced Self-Motion Illusion (Circular Vection) in Virtual Reality. Proceedings of 8th Annual Workshop Presence 2005 (pp. 49-57). Retrieved from http://en.scientificcommons.org/20596230

Riecke BE, Schulte-Pelkum J, Caniard F, Bülthoff HH (2005b) Towards Lean and Elegant Self-Motion Simulation in Virtual Reality. Proceedings of the 2005 IEEE Conference 2005 on Virtual Reality, VR '05 (pp. 131-138). doi:10.1109/VR.2005.83

Riecke BE, Väljamäe A, Schulte-Pelkum J (2009) Moving sounds enhance the visually-induced self-motion illusion (circular vection) in virtual reality. ACM Transactions on Applied Perception (TAP), 6, 7:1-7:27 doi:http://doi.acm.org/10.1145/1498700.1498701

Rieser JJ, Pick HL, Ashmead DH, Garing AE (1995) Calibration of human locomotion and models of perceptual-motor organization. J. Exp. Psychol. Hum. Percept. Perf. 21:480-497

Schulte-Pelkum J (2007) Perception of self-motion: Vection experiments in multi-sensory Virtual Environments (PhD thesis). Ruhr-Universität 
Bochum.

Seno T, Abe K, Kiyokawa S (2013) Wearing heavy iron clogs can inhibit vecton. Multisensory Research, 26:569-580. doi: 10.1163/22134808-00002433

Seno T, Hasuo E, Ito H, Nakajima Y (2012) Perceptually plausible sounds facilitate visually induced self-motion perception (vection). Perception 41:577-593. doi:10.1068/p7184

Seno T, Fukuda H (2012) Stimulus meanings alter illusory self-motion (vection)-Experimental examination of the train illusion. Seeing and Perceiving 25:631-645 doi:10.1163/18784763-00002394

Seno T, Ito H, Sunaga S (2011) Inconsistent locomotion inhibits vection. Perception 40:747-750 doi:10.1068/p7018

Seno T, Ogawa M, Ito H, Sunaga S (2011) Consistent air flow to the face facilitates vection. Perception 40:1237-1240 doi:10.1068/p7055

Wallach H, Flaherty EW (1975) A compensation for field expansion caused by moving forward. Perception \& Psychophysics 17:445-449 doi: 10.3758/BF03203291

Wong SCP, Frost BJ (1981) The effect of visual-vestibular conflict on the latency of steady-state visually induced subjective rotation. Perception \& Psychophysics 30:228-236 doi: 10.3758/BF03214278

Wright WG (2009) Linear vection in virtual environments can be strengthened by discordant inertial input. 31st Annual International Conference of the IEEE EMBS (Engineering in Medicine and Biology Society) (pp. 1157-1160). Minneapolis, USA. doi:10.1109/IEMBS.2009.5333425 